\title{
Evaluating the Relationship Between Myofascial Pain Syndrome (MPS) and Physical and Mental Health Status in Patients with Stroke
}

\author{
Masoud Hatefi (iD ${ }^{1}$ and Lida Nouri (iD) ${ }^{2,}$ \\ ${ }^{1}$ School of Medicine, Non-communicable Diseases Research Center, Emam Khomeini Hospital, Ilam University of Medical sciences, Ilam, Iran \\ ${ }^{2}$ Department of Anesthesiology, School of Allied Medical Sciences, Ilam University of Medical sciences, Ilam, Iran \\ Corresponding author: Department of Anesthesiology, School of Allied Medical Sciences, Ilam University of Medical sciences, Ilam, Iran. Email: lidanouri2016@gmail.com \\ Received 2021 September 13; Revised 2021 October 16; Accepted 2021 October 21.
}

\begin{abstract}
Background: Myofascial pain syndrome (MPS) is a non-inflammatory disorder with muscle stiffness and pain that occurs with the appearance of palpable and irritating nodules in the muscular system. Stroke is one of the most common neurological diseases that in many cases leads to disability and reduction of quality of life (QOL).

Objectives: This study aimed to evaluate the relationship between MPS and physical and mental health (MH) status in patients with stroke.

Methods: Using available sampling method, this case-control study included 260 patients with stroke. To collect data, demographic characteristics, Visual Analogue Scale (VAS), 36-item Short Form Survey (SF-36), and Depression, Anxiety and Stress Scale (DASS-21) were used. Data analysis was performed using SPSS 16. Mean and standard deviation were used for descriptive statistics and independent $t$-test, paired $t$-test, and analysis of variance (ANOVA) were used for inferential tests.

Results: While the pain score was 6.35 (1.39), QOL score was 38.86 (11.69), and MH score was 16.26 (2.75) in the intervention group, these scores were 2.15 (0.96), 63.96 (17.52), and 9.02 (4.63), respectively, in the control group. The results showed no statistically significant relationship between MPS and QOL. But there was a statistically significant relationship between MPS and MH, so that the MH status of patients with MPS was lower than the MH status of other patients.

Conclusions: Necessary interventions have been done to improve the health status of patients with MPS, which will lead to an increase in the health status of these patients.
\end{abstract}

Keywords: Myofascial Pain Syndrome, Pain, Stroke

\section{Background}

Pain is an unpleasant feeling leading to an unpleasant experience in a person's health. There are various factors affecting pain, such as pain syndromes. These types of syndromes include fibromyalgia syndrome (1), complex regional pain syndrome (2), patellofemoral pain syndrome (3), complex regional pain syndrome (4), and myofascial pain syndrome (MPS) (5).

MPS is a non-inflammatory disorder with muscle stiffness and pain that occurs with the appearance of palpable and irritating nodules in the muscular system (6). It is one of the most common causes of musculoskeletal problems, the most important cause of illness in adults (7), and one of the main causes of pain and dysfunction in the musculoskeletal system. This syndrome is a common, non-joint, and musculoskeletal disorder, one of the important features of which is the presence of trigger points $(6,8)$. Other symptoms of MPS include diffuse pain, decreased range of motion, and the need for symptoms of the autonomic system, whose chronic and diffuse pain may be due to central and peripheral pain mechanisms (9). Patients with MPS are more likely to experience stress, anxiety, depression, pain, decreased sleep, and disability $(10,11)$.

Myofascial Trigger Points (MTrPs) are local and irritating points associated with palpable nodules in taut bands (TB) with two latent and active types $(12,13)$. Patients with this syndrome may experience problems that affect their health (14). Among the factors affecting health, we can mention the quality of life (QoL) and mental health (MH) (15).

QOL is one of the most important factors affecting health. The World Health Organization (WHO) defines QOL as an individual's understanding of his/her position in cultural systems and values. In fact, QOL is a wide range of human experiences, including daily needs such as food and 
shelter, interpersonal and interpersonal responses to illness, and activities related to professional success and happiness $(16,17)$. QOL is also defined as an individual's personal perception of his/her place in the culture and value system of the society in which s/he lives and his/her relationship with goals, expectations, standards, and concerns (18). QOL is affected by demographic and social variables, diseases, and clinical status of individuals, and it has been presented as a major issue in patient care, especially in chronic patients $(19,20)$.

Emotional and mental health problems such as depression, anxiety, and stress reduce mental well-being and life satisfaction. Naturally, more behavioral, cognitive, and emotional problems lead to less satisfaction felt by a person $(21,22)$. Mental health problems are defined in five areas: emotional, behavioral, hyperactivity, peer communication, and social cohesion (23). Maintaining and promoting health in all age groups is an unavoidable health necessity, and mental health includes the "feeling of well-being" that enables a person to identify their abilities and adapt to the usual stresses of life and work to build community (24).

One of the diseases that affects the MH and QOL status of patients is stroke (25). Stroke is one of the most common neurological diseases that in many cases leads to disability and reduction of QOL (26). Factors related to patients with stroke are effective in management and planning to improve the health of these patients, and they are very important for the health care system. Hence, it is essential to pay special attention to these patients (27).

\section{Objectives}

Given the significant prevalence of stroke and the importance of paying attention to pain in these patients, the aim of this study was to evaluate the relationship between MPS and physical and MH status in patients with stroke.

\section{Methods}

\subsection{Study Design}

Using available sampling method, this case-control study included patients with stroke living in Ilam, Iran.

\subsection{Study Population}

A total of 280 patients with stroke (140 patients with MPS and 140 patients without MPS) were included in the study.

\subsection{Inclusion and Exclusion Criteria}

\subsubsection{Inclusion Criteria}

The inclusion criteria were: Living in Ilam; having a stroke; conscious consent to participate in the study; and being in the age range of 20 - 65 years.

The diagnosis of active trigger points in the studied muscles was made by an expert with a bachelor's degree in physiotherapy with at least five years of experience based on the presence of a tight band in the muscle, having sensitive points in the muscle, and pain reset (special pattern of pain propagation following pressure on the trigger points).

\subsubsection{Exclusion Criteria}

The exclusion criteria were: Reluctance to participate in the study; having blood coagulation problems; consuming drugs or corticosteroids; pregnancy; having mental illness or psychological problems; patients with trauma leading to pain, fractures, depression; the use of muscle relaxants; and people with chronic pain.

\subsection{Data Gathering}

\subsubsection{Demographic Characteristic}

The demographic characteristics form included information on age, gender, marriage, education (illiterate, literate), length of stroke (less than 5 years, between 5 and 10 years, more than 10 years), economic status (weak, moderate, high).

\subsubsection{Visual Analogue Scale (VAS)}

Visual Analogue Scale (VAS) was used to measure pain, which is a scale from 0 to $10(28,29)$. MPS was based on the presence of tight band, pain, and tenderness (10).

\subsubsection{6-Item Short Form Survey (SF-36)}

To measure the QOL, the Persian version of 36-item short form survey (SF-36) was used, which has 36 items in eight dimensions of health, with a total score between zero (lowest score) to 100 (highest score). The dimensions of this questionnaire include physical problems, limitations in playing the role due to physical problems, physical pain, general health, vitality, social functioning, limitations in playing the role due to emotional problems, and mental health (30). 


\subsubsection{Depression, Anxiety and Stress Scale (DASS-21)}

To assess stress, anxiety, and depression, the Persian version of Depression, Anxiety and Stress Scale (DASS21) was used. This scale includes questions on stress (seven questions), anxiety (seven questions), and depression (seven questions). The overall scoring of this questionnaire is as follows: a score between $0-4$ is considered as a normal score, a score of 5 - 11 is considered as a moderate score, and a score above 12 is considered as a severe score (31).

\subsection{Research Method}

Sampling was done after obtaining the necessary permits from the relevant authorities of Ilam University of Medical Sciences. We obtained an informed consent from all patients by explaining the objectives of the research and assuring the confidentiality of their information. Participation in the study was completely voluntary, and nonparticipation in the study had no effect on providing services to patients. Sampling method was of available type, and patients referred to hospitals in Ilam were included in the study. To evaluate all muscles based on the main trigger points, the patient was lying on his/her side. We examined the myofascial status of the multifidus muscle, quadratus lumborum muscle and Iliopsoas muscle, Pectoralis major muscle, Iliocostalis muscle, Piriformis muscle, Gluteus maximus muscle, Gluteus medius and Minimus muscle, Fascia Lata tensor muscle, Bapps femoris muscle, venomous membranous muscle, gastrocnemius and solius muscle, peroneal muscle of upper upper and lower third of leg, and anterior tibialis muscle (32). To collect the samples, purpose-based sampling method was used. Ethical criteria in the research, including informed written satisfaction of patients, voluntary participation in the study, free examination of patients, and all other ethical guidelines were observed.

\subsection{Data Analysis}

Data analysis was performed using SPSS 16. Mean and standard deviation were used for descriptive statistics and independent $t$-test, paired $t$-test, and analysis of variance (ANOVA), and linear regression were used for inferential tests.

\section{Results}

Table 1 shows the demographic characteristics of all patients. According to the findings, no statistically significant relationship was observed between any of the demographic characteristics $P>0.05$. The findings also showed that most of the patients were male, unemployed, single, with moderate social support, and poor economic status (Table 1).

According to the findings, while the pain score was 6.35 (1.39), QOL score was 38.86 (11.69), and MH score was 16.26 (2.75) in the intervention group, these scores were 2.15 (0.96), 63.96 (17.52), and 9.02 (4.63), respectively, in the control group. A statistically significant difference was observed between the control and case groups in all the mean scores obtained in the variables of pain, QOL, and $\mathrm{MH}(\mathrm{P}<$ 0.001).

Table 2 compares the mean pain, QOL, and MH scores in the control and case groups according to the demographic characteristics. According to the findings, in patients with fibromyalgia, there was a statistically significant relationship between pain status and family support and marital status $\mathrm{P}<0.05$ (Table 2 ).

According to the findings of Tables 3 and 4, there was no statistically significant relationship between MPS and QOL. But there was a statistically significant inverse relationship between MPS and MH, so that the MH status of patients with MPS was lower than the MH status of other patients. According to the findings, there was no relationship between age and pain status, but the QOL and health of patients decreased in older ages $(\mathrm{P}<0.05)$.

\section{Discussion}

The aim of this study was to investigate the relationship between MPS and physical and mental health in patients with stroke. According to the findings, a high prevalence of pain was reported in patients with stroke. A study by Westerlind et al. showed that $40 \%$ of patients with stroke experienced pain five years after stroke (33). According to Haslam et al., 58\% of study participants had moderate to severe pain. Also, 90.1\% of stroke patients used analgesics to relieve pain. The most common of these are anti-inflammatory, anti-seizure, and anti-depressant drugs (34). According to Khazaal et al., in the analyses performed three months after stroke, neuropathic pain was reported at $24.8 \%$, and cognitive impairment was reported at $69.2 \%$ (35).

According to the findings of this study, the QOL of patients was reported to be poor. Schindel et al. reported that the QOL level of patients with stroke was low; also, with the decrease of social support, the QOL level of patients was further reduced (36). In a meta-analysis by Bello et al. on 2,752 patients with stroke, it was shown that these patients had a lower QOL than the population of healthy individuals (37). This is consistent with the results of this 


\begin{tabular}{|c|c|c|c|}
\hline Variable & Cases & Control & P-Value \\
\hline Sex & - & - & 0.45 \\
\hline Male & $74(56.9)$ & $71(54.6)$ & - \\
\hline Female & $56(43.1)$ & $59(45.4)$ & - \\
\hline Job status & - & - & 0.46 \\
\hline Employed & $54(41.5)$ & $79(60.8)$ & - \\
\hline Unemployed & $76(58.5)$ & $51(39.2)$ & - \\
\hline Education & - & - & 0.19 \\
\hline Primary school & $38(29.2)$ & $32(24.6)$ & - \\
\hline Associate diploma & $84(64.6)$ & $91(70)$ & - \\
\hline Postgraduate & $8(6.2)$ & $7(5.4)$ & - \\
\hline Marital status & - & - & 0.25 \\
\hline Single & $95(73.1)$ & $99(76.2)$ & - \\
\hline Married & $35(26.9)$ & $31(23.8)$ & - \\
\hline Family support & - & - & 0.43 \\
\hline Low & $33(25.4)$ & $45(34.6)$ & - \\
\hline Medium & $54(41.5)$ & $47(36.2)$ & - \\
\hline Much & $43(33.1)$ & $38(29.2)$ & - \\
\hline Income & . & - & 0.54 \\
\hline Low & $69(53.1)$ & $71(54.6)$ & - \\
\hline Medium & $56(43.1)$ & $52(40)$ & - \\
\hline High & $5(3.8)$ & $7(5.4)$ & - \\
\hline Age (M(SD)) & $69.43(13.12)$ & $70.70(14.70)$ & 0.76 \\
\hline
\end{tabular}

${ }^{\mathrm{a}}$ Values are expressed as No. (\%).

study, which showed that patients with stroke reported lower QOL than healthy individuals in physical and mental dimensions.

According to our findings, lower mental health was reported in patients with MPS. Westerlind et al. demonstrated that the patients who reported more pain had more restricted mobility and depression and less recovery after stroke compared with patients with less pain, which is consistent with the results of this study (33). Khazaal et al. also showed that $94 \%$ of patients reported fatigue and cognitive impairment at $69.2 \%$, anxiety at $51.3 \%$, and depression at $76.1 \%$. Also, patients with neuropathic pain experienced more pain than patients without neuropathic pain (35).

According to the findings of this study, MPS could affect the health status of stroke patients. In a study by IglesiasGonzález et al. (38), MPS had negative effects on the health status of patients with low back pain. Furthermore, Ezzati et al. (10) showed that this syndrome had negative effects on the health status of patients with neck pain, which is consistent with the results of our study.

\subsection{Conclusions}

In conclusion, patients with MPS had a lower mental health. Necessary interventions have been done to improve the health status of patients with MPS, which will lead to an increase in the health status of these patients.

\section{Acknowledgments}

The authors thank the Student Research Committee, Ilam University of Medical Sciences, Ilam, Iran, for sincere collaboration.

\section{Footnotes}

Authors' Contribution: MH \& LN did study conception, data analysis, and manuscript writing. MH \& LN did data 
Hatefi M and Nouri L

\begin{tabular}{|c|c|c|c|c|c|c|c|c|c|}
\hline \multirow{2}{*}{ Variable } & \multicolumn{3}{|c|}{ Pain } & \multicolumn{3}{|c|}{ QOL } & \multicolumn{3}{|c|}{ MH } \\
\hline & Cases & Control & P-Value & Cases & Control & P-Value & Cases & Control & P-Value \\
\hline Sex & & & $<0.05$ & & & $<0.05$ & & & $<0.05$ \\
\hline Male & $6.55(1.44)$ & $2.28(1.0)$ & & $39.45(12.33)$ & $65.18(18.10)$ & & $16.35(2.83)$ & $8.38(4.59)$ & \\
\hline Female & $6.10(1.28)$ & $2(0.9)$ & & $\begin{array}{c}38.08 \\
(10.84)\end{array}$ & $62.50(16.84)$ & & $16.16(2.65)$ & $9.79(4.60)$ & \\
\hline P-value & 0.07 & 0.09 & - & 0.51 & 0.38 & - & 0.69 & 0.08 & - \\
\hline Job status & & & $<0.05$ & & & $<0.05$ & & & $<0.05$ \\
\hline Employed & $6.42(1.35)$ & $2.07(0.81)$ & & $\begin{array}{c}39.44 \\
(10.47)\end{array}$ & $62.10(17.61)$ & & $16.11(2.85)$ & $9.26(4.82)$ & \\
\hline Unemployed & $6.31(1.42)$ & $2.27(1.16)$ & & $\begin{array}{c}38.46 \\
(12.54)\end{array}$ & 66.86(17.15) & & $16.38(2.68)$ & $8.64(4.34)$ & \\
\hline P-value & 0.65 & 0.25 & - & 0.63 & 0.13 & - & 0.58 & 0.45 & - \\
\hline Education & & & $<0.05$ & & & $<0.05$ & & & $<0.05$ \\
\hline Primary school & $6.42(1.30)$ & $2.03(0.89)$ & & $40(10.36)$ & $64.40(14.70)$ & & $15.5(2.39)$ & $9.65(5.09)$ & \\
\hline $\begin{array}{l}\text { Associate } \\
\text { diploma }\end{array}$ & $6.42(1.30)$ & $2.20(0.97)$ & & $\begin{array}{c}38.78 \\
(12.40)\end{array}$ & 64.43(18.35) & & $16.60(2.79)$ & $8.87(4.51)$ & \\
\hline Postgraduate & $6.39(1.42)$ & $2.0(1.29)$ & & $34.37(0.84)$ & $55.85(7.12)$ & & $16.37(3.46)$ & $8(4.28)$ & \\
\hline P-value & 0.44 & 0.61 & - & 0.46 & 0.45 & - & 0.11 & 0.60 & - \\
\hline Family support & & & $<0.05$ & & & $<0.05$ & & & $<0.05$ \\
\hline Low & $5.96(1.31)$ & $2.20(0.86)$ & & $38.12(11.78)$ & 65.35(16.16) & & $16.15(2.71)$ & $9.24(5.15)$ & \\
\hline Medium & $6.29(1.31)$ & $2.0(1.12)$ & & $\begin{array}{c}37.50 \\
(12.44)\end{array}$ & 63.04(19.69) & & $16.11(2.56)$ & $9.27(4.76)$ & \\
\hline High & $6.74(1.48)$ & $2.28(0.86)$ & & $41.16(10.51)$ & $63.47(16.56)$ & & $16.55(3.02)$ & $8.44(3.82)$ & \\
\hline P-value & 0.04 & 0.36 & - & 0.24 & 0.80 & - & 0.70 & 0.66 & - \\
\hline Marital status & & & $<0.05$ & & & $<0.05$ & & & $<0.05$ \\
\hline Single & $6.56(1.36)$ & $2.10(0.87)$ & & $38.18(11.07)$ & $64.59(16.68)$ & & 16.47 & $9.04(4.76)$ & \\
\hline Married & $5.80(1.32)$ & $2.32(1.22)$ & & $40.71(13.21)$ & $61.96(20.16)$ & & 16.71 & $8.96(4.27)$ & \\
\hline P-value & 0.04 & 0.26 & - & 0.27 & 0.46 & - & 0.16 & 0.94 & - \\
\hline Income & & & $<0.05$ & & & $<0.05$ & & & $<0.05$ \\
\hline Low & $6.46(1.33)$ & $1.91(0.85)$ & & $39.53(12.17)$ & $60.56(18.12)$ & & $16.47(2.84)$ & $9.19(4.76)$ & \\
\hline Medium & $6.30(1.46)$ & $2.40(1.05)$ & & $38.46(11.38)$ & $68.11(16.44)$ & & $15.98(2.70)$ & $8.90(4.56)$ & \\
\hline High & $5.60(1.39)$ & $2.71(0.75)$ & & $34.20(8.46)$ & 67.71(13.08) & & $16.6(1.94)$ & $8.14(4.25)$ & \\
\hline P-value & 0.37 & 0.006 & - & 0.58 & 0.05 & - & 0.58 & 0.82 & - \\
\hline
\end{tabular}

${ }^{\mathrm{a}}$ Values are expressed as No. (\%).

\begin{tabular}{|c|c|c|c|c|c|c|}
\hline Mode & & Sum of Squares & Df & Mean Square & $\mathbf{F}$ & P-Value \\
\hline \multirow[t]{4}{*}{ QOL } & & & & & 0.38 & 0.53 \\
\hline & Regression & 0.752 & 1 & 0.752 & & \\
\hline & Residual & 249.256 & 128 & 1.94 & & \\
\hline & Total & 250.008 & 129 & & & \\
\hline \multirow[t]{4}{*}{ MH } & & & & & 7.939 & 0.006 \\
\hline & Regression & 14.600 & 1 & 14.600 & & \\
\hline & Residual & 235.408 & 128 & 1.839 & & \\
\hline & Total & 250.008 & 129 & & & \\
\hline
\end{tabular}


Hatefi $M$ and Nouri L

\begin{tabular}{|c|c|c|c|c|c|}
\hline \multirow{2}{*}{ Model } & \multicolumn{2}{|c|}{ Unstandardized Coefficients } & \multirow{2}{*}{$\begin{array}{l}\text { Standardized } \\
\text { Coefficients } \\
\text { Beta }\end{array}$} & \multirow{2}{*}{$\mathbf{T}$} & \multirow{2}{*}{ P-Value } \\
\hline & B & Std. Error & & & \\
\hline \multirow{2}{*}{ QOL (Constant) } & 6.108 & 0.426 & & 14.32 & 0.000 \\
\hline & 0.007 & 0.011 & 0.055 & 0.621 & 0.535 \\
\hline \multirow{2}{*}{ MH(Constant) } & 4.371 & 0.716 & & 6.102 & 0.000 \\
\hline & 0.122 & 0.043 & 0.242 & 2.818 & 0.006 \\
\hline
\end{tabular}

collection and manuscript writing. MH \& LN did data collection and manuscript writing. MH \& LN did data collection and manuscript writing. MH \& LN did data collection and manuscript writing. MH \& LN did result interpretation and manuscript writing. MH \& LN did study design, manuscript writing, and manuscript editing.

Conflict of Interests: The authors declare no conflict of interest.

Ethical Approval: The Ethics Committee of the Ilam University of Medical Sciences, Iran, approved the study (IR.MEDILAM.REC.1400.289).

Funding/Support: Ilam University of Medical Sciences, Ilam, Iran (Project Code: A-10-3340-1).

Informed Consent: Informed consent was obtained from all participants.

\section{References}

1. D’Onghia M, Ciaffi J, McVeigh JG, Di Martino A, Faldini C, Ablin JN, et al. Fibromyalgia syndrome - a risk factor for poor outcomes following orthopaedic surgery: A systematic review. Semin Arthritis Rheum. 2021;51(4):793-803. doi: 10.1016/j.semarthrit.2021.05.016. [PubMed: 34153893].

2. Harden RN, Oaklander AL, Burton AW, Perez RS, Richardson K, Swan $\mathrm{M}$, et al. Complex regional pain syndrome: practical diagnostic and treatment guidelines, 4th edition. Pain Med. 2013;14(2):180-229. doi: 10.1111/pme.12033. [PubMed: 23331950].

3. Albornoz-Cabello M, Barrios-Quinta CJ, Barrios-Quinta AM, EscobioPrieto I, Cardero-Duran MLA, Espejo-Antunez L. Effectiveness of TelePrescription of Therapeutic Physical Exercise in Patellofemoral Pain Syndrome during the COVID-19 Pandemic. Int J Environ Res Public Health. 2021;18(3). doi: 10.3390/ijerph18031048. [PubMed: 33504042]. [PubMed Central: PMC7908506].

4. Taylor SS, Noor N, Urits I, Paladini A, Sadhu MS, Gibb C, et al. Complex Regional Pain Syndrome: A Comprehensive Review. Pain Ther. 2021;10(2):875-92. doi: 10.1007/s40122-021-00279-4. [PubMed: 34165690]. [PubMed Central: PMC8586273].

5. Galasso A, Urits I, An D, Nguyen D, Borchart M, Yazdi C, et al. A Comprehensive Review of the Treatment and Management of Myofascial Pain Syndrome. Curr Pain Headache Rep. 2020;24(8):43. doi: 10.1007/s11916020-00877-5. [PubMed: 32594264].

6. Badil Guloglu S, Tunc S. The assessment of affective temperament and life quality in myofascial pain syndrome patients. Int $J$ Psychiatry Clin Pract. 2020:1-6. doi: 10.1080/13651501.2020.1833039. [PubMed: 33084454].

7. Ay S, Konak HE, Evcik D, Kibar S. The effectiveness of Kinesio Taping on pain and disability in cervical myofascial pain syndrome. Rev
Bras Reumatol Engl Ed. 2017;57(2):93-9. doi: 10.1016/j.rbre.2016.03.012. [PubMed: 28343625].

8. Karayanni H, Dror AA, Oren D, Sela E, Granot I, Srouji S. Exacerbation of chronic myofascial pain during COVID-19. Oral Maxillofac Surg. 2021;1. doi: 10.1016/j.adoms.2021.100019.

9. Fernandez-de-Las-Penas C, Cuadrado ML, Arendt-Nielsen L, Ge HY, Pareja JA. Increased pericranial tenderness, decreased pressure pain threshold, and headache clinical parameters in chronic tension-type headache patients. Clin J Pain. 2007;23(4):346-52. doi: 10.1097/AJP.ob013e31803b3770. [PubMed: 17449996].

10. Ezzati K, Ravarian B, Saberi A, Salari A, Reyhanian Z, Khakpour M, et al. Prevalence of Cervical Myofascial Pain Syndrome and its Correlation with the Severity of Pain and Disability in Patients with Chronic Non-specific Neck Pain. Arch BoneJt Surg. 2021;9(2):230-4. doi: 10.22038/abjs.2020.48697.2415. [PubMed: 34026942]. [PubMed Central: PMC8121028].

11. Munoz-Munoz S, Munoz-Garcia MT, Alburquerque-Sendin F, ArroyoMorales M, Fernandez-de-las-Penas C. Myofascial trigger points, pain, disability, and sleep quality in individuals with mechanical neck pain. J Manipulative Physiol Ther. 2012;35(8):608-13. doi: 10.1016/j.jmpt.2012.09.003. [PubMed: 23158466].

12. Castaldo M, Ge HY, Chiarotto A, Villafane JH, Arendt-Nielsen L. Myofascial trigger points in patients with whiplash-associated disorders and mechanical neck pain. Pain Med. 2014;15(5):842-9. doi: 10.1111/pme.12429. [PubMed: 24641263].

13. Villafane JH, Herrero P. Conservative treatment of Myofascial Trigger Points and joint mobilization for management in patients with thumb carpometacarpal osteoarthritis. J Hand Ther. 2016;29(1):89-92. quiz 92. doi: 10.1016/j.jht.2015.10.005. [PubMed: 26704595].

14. Ginszt M, Zieliński G, Berger M, Szkutnik J, Bakalczuk M, Majcher P. Acute Effect of the Compression Technique on the Electromyographic Activity of the Masticatory Muscles and Mouth Opening in Subjects with Active Myofascial Trigger Points. Appl Sci. 2020;10(21). doi: 10.3390/app10217750.

15. Castro Sanchez AM, Garcia Lopez H, Fernandez Sanchez M, Perez Marmol JM, Aguilar-Ferrandiz ME, Luque Suarez A, et al. Improvement in clinical outcomes after dry needling versus myofascial release on pain pressure thresholds, quality of life, fatigue, pain intensity, quality of sleep, anxiety, and depression in patients with fibromyalgia syndrome. Disabil Rehabil.2019;41(19):2235-46. doi: 10.1080/09638288.2018.1461259. [PubMed: 29681188].

16. Sadeghizadeh M, Bagherian B, Vahidi H, Sabzevari S. [Effect of applying the specific situation theory on the quality of life in patients with heart failure].J Hayat. 2021;27(2):146-60. Persian.

17. Mokhatri-Hesari P, Montazeri A. Health-related quality of life in breast cancer patients: review of reviews from 2008 to 2018. Health Qual Life Outcomes. 2020;18(1):338. doi: 10.1186/s12955-020-01591-x. [PubMed: 33046106]. [PubMed Central: PMC7552560].

18. Maghzi Najafabadi A, Roohafza H, Feizi A, Sarrafzadegan N. Association between shiftwork and second job with quality of life: a crosssectional study in a large sample of Isfahan steel company's employees. Koomesh J. 2020;22(1):122-9. doi: 10.29252/koomesh.22.1.122. 
19. Silva DS, Andrade Edos S, Elias RM, David-Neto E, Nahas WC, Castro MC, et al. The perception of sleep quality in kidney transplant patients during the first year of transplantation. Clinics (Sao Paulo). 2012;67(12):1365-71. doi: 10.6061/clinics/2012(12)04. [PubMed: 23295588]. [PubMed Central: PMC3521797].

20. Sotodeh Asl N, Avazabadian M, Ghorbani R, Malek F. Quality of life in patients with hypertension and type 2 diabetes mellitus. Koomesh $\mathrm{J}$. 2020;22(2):263-8. doi: 10.29252/koomesh.22.2.263.

21. O'Connor RC, Wetherall K, Cleare S, McClelland H, Melson AJ, Niedzwiedz CL, et al. Mental health and well-being during the COVID-19 pandemic: longitudinal analyses of adults in the UK COVID-19 Mental Health \& Wellbeing study. Br J Psychiatry. 2020:18. doi: 10.1192/bjp.2020.212. [PubMed: 33081860]. [PubMed Central: PMC7684009].

22. Pierce M, Hope H, Ford T, Hatch S, Hotopf M, John A, et al. Mental health before and during the COVID-19 pandemic: a longitudinal probability sample survey of the UK population. Lancet Psychiatry. 2020;7(10):883-92. doi: 10.1016/S2215-0366(20)30308-4. [PubMed: 32707037]. [PubMed Central: PMC7373389].

23. Goodman A, Lamping DL, Ploubidis GB. When to use broader internalising and externalising subscales instead of the hypothesised five subscales on the Strengths and Difficulties Questionnaire (SDQ): data from British parents, teachers and children. J Abnorm Child Psychol. 2010;38(8):1179-91. doi: 10.1007/s10802-010-9434-x. [PubMed: 20623175].

24. Radez J, Reardon T, Creswell C, Lawrence PJ, Evdoka-Burton G, Waite P. Why do children and adolescents (not) seek and access professional help for their mental health problems? A systematic review of quantitative and qualitative studies. Eur Child Adolesc Psychiatry. 2021;30(2):183-211. doi: 10.1007/s00787-019-01469-4. [PubMed: 31965309]. [PubMed Central: PMC7932953].

25. Alquwez N, Alshahrani AM. Influence of Spiritual Coping and Social Support on the Mental Health and Quality of Life of the Saudi Informal Caregivers of Patients with Stroke. J Relig Health. 2021;60(2):787803. doi: 10.1007/s10943-020-01081-w. [PubMed: 32889677].

26. Pedersen SG, Friborg O, Heiberg GA, Arntzen C, Stabel HH, Thrane G, et al. Stroke-Specific Quality of Life one-year post-stroke in two Scandinavian country-regions with different organisation of rehabilitation services: a prospective study. Disabil Rehabil. 2021;43(26):3810-20. doi: 10.1080/09638288.2020.1753830. [PubMed: 32356473].

27. Caro JJ, Huybrechts KF, Duchesne I. Management patterns and costs of acute ischemic stroke : an international study. For the Stroke Economic Analysis Group. Stroke. 2000;31(3):582-90. doi: 10.1161/01.str.31.3.582. [PubMed:10700489].

28. Huang Z, Kohler IV, Kampfen F. A Single-Item Visual Analogue Scale
(VAS) Measure for Assessing Depression Among College Students. Community Ment Health J. 2020;56(2):355-67. doi: 10.1007/s10597-01900469-7. [PubMed: 31531784].

29. Heller GZ, Manuguerra M, Chow R. How to analyze the Visual Analogue Scale: Myths, truths and clinical relevance. Scand J Pain. 2016;13:67-75. doi: 10.1016/j.sjpain.2016.06.012. [PubMed: 28850536].

30. Hobart JC, Williams LS, Moran K, Thompson AJ. Quality of life measurement after stroke: uses and abuses of the SF-36. Stroke. 2002;33(5):1348-56. doi: 10.1161/01.str.0000015030.59594.b3. [PubMed: 11988614].

31. Norton PJ. Depression Anxiety and Stress Scales (DASS-21): psychometric analysis across four racial groups. Anxiety Stress Coping. 2007;20(3):253-65. doi: 10.1080/10615800701309279. [PubMed: 17999228].

32. Yousefzadeh Chabok S, Ezzati K, Saberi A, Hosein Zadeh J. [Evaluating the Prevalence of Lumbar Myofascial Pain Syndrome in Patients with Non-specific Chronic Low Back Pain and a Normal MRI Study]. Jour of gums. 2020;29(1):43-50. Persian.

33. Westerlind E, Singh R, Persson HC, Sunnerhagen KS. Experienced pain after stroke: a cross-sectional 5-year follow-up study. BMC Neurol. 2020;20(1):4. doi: 10.1186/s12883-019-1584-z. [PubMed: 31910805]. [PubMed Central: PMC6945579].

34. Haslam BS, Butler DS, Kim AS, Carey LM. Chronic pain following stroke: Current treatment and perceived effect. Disabil Health J. 2021;14(1):100971. doi: 10.1016/j.dhjo.2020.100971. [PubMed: 32830081].

35. Khazaal W, Taliani M, Boutros C, Abou-Abbas L, Hosseini H, Salameh P, et al. Psychological Complications at 3 Months Following Stroke: Prevalence and Correlates Among Stroke Survivors in Lebanon. Front Psychol. 2021;12:663267. doi: 10.3389/fpsyg.2021.663267. [PubMed: 34177717]. [PubMed Central: PMC8222528].

36. Schindel D, Schneider A, Grittner U, Jobges M, Schenk L. Quality of life after stroke rehabilitation discharge: a 12-month longitudinal study. Disabil Rehabil. 2021;43(16):2332-41. doi: 10.1080/09638288.2019.1699173. [PubMed: 31846594].

37. Bello UM, Chutiyami M, Salihu D, Abdu SI, Tafida BA, Jabbo AA, et al. Quality of life of stroke survivors in Africa: a systematic review and meta-analysis. Qual Life Res. 2021;30(1):1-19. doi: 10.1007/s11136-02002591-6. [PubMed: 32712933].

38. Iglesias-Gonzalez JJ, Munoz-Garcia MT, Rodrigues-de-Souza DP, Alburquerque-Sendin F, Fernandez-de-Las-Penas C. Myofascial trigger points, pain, disability, and sleep quality in patients with chronic nonspecific low back pain. Pain Med. 2013;14(12):1964-70. doi: 10.1111/pme.12224. [PubMed: 23947760]. 